\title{
Impacto económico de la devolución del impuesto a la renta y el IVA en el sector florícola
}

Fecha de recepción: 17/01/2020 • Fecha de aceptación: 20/03/2020 • Fecha de publicación:10/06/2020

\author{
María Sonia Bastidas Gutiérrez ${ }^{1}$ \\ Rosentantau Del Ecuador S.A. \\ soniabastidasg@yahoo.com \\ Marlene Del Pilar Chicaiza Quilo² \\ Punto Gráfico Celin, Ecuador \\ marcelinaqu@hotmail.com \\ Miguel Angel Aizaga Villate ${ }^{3}$ \\ Universidad Israel, Ecuador \\ maizaga@uisrael.edu.ec \\ https://orcid.org/0000-0002-4492-1181
}

\section{RESUMEN:}

El objetivo del presente artículo es evidenciar la importancia del sector florícola en la economía del Ecuador y develar las implicaciones tributarias que se generan por mantener operaciones internacionales, pretendiendo exponer las circunstancias económicas actuales y la relevancia de la devolución del impuesto al valor agregado (IVA) y del impuesto a la renta (IR). Para ello se describen de manera sucinta estos impuestos y el derecho a la devolución de los mismos establecida acorde a la base legal. En cuanto a la metodología el estudio es de tipo documental, con un nivel descriptivo y enfoque cuantitativo, puesto que se recolectan datos provenientes de estadísticas para determinar las características del sector. Una de las principales conclusiones es que al analizar los datos desde el 2015 hasta el 2018, el sector florícola es uno de los más importantes en el comercio exterior del país, puesto que ocupa el tercer lugar en el ranking de exportación mundial, también es gran generador de empleos y su aportación al PIB tiene tendencia creciente; sin embargo, al revisar la situación económica, los beneficios han disminuido, por lo que se hace pertinente la investigación para analizar los 
factores incidentes.

PALABRAS CLAVE: sector florícola, exportación, implicaciones tributarias, impacto económico.

\begin{abstract}
:
The objective of this article is to demonstrate the importance of the floriculture sector in the Ecuadorian economy and reveal the tax implications generated by maintaining international operations, seeking to expose the current economic circumstances and the relevance of the return of the value added tax (VAT) and income tax (IR). To do this, these taxes and the right to their refund established according to the legal basis are briefly described. As for the methodology, the study is of a documentary type, with a descriptive level and a quantitative approach, since data from statistics are collected to determine the characteristics of the sector. One of the main conclusion is that when analyzing the data from 2015 to 2018, the flower sector is one of the most important in the country's foreign trade, since it occupies third place in the world export ranking, it is also a great generator of jobs and their contribution to GDP has an increasing trend, however, when reviewing the economic situation, the benefits have decreased, reason why the investigation becomes relevant to analyze the incident factors.
\end{abstract}

KEYWORDS: floriculture sector, export, tax implications, economic impact. 


\section{Introducción}

Las entidades del sector florícola al realizar sus compras cancelan la tarifa de Impuesto a la Renta y el Valor Agregado sobre lo que determina la ley para la transferencia de bienes muebles de naturaleza corporal y la adquisición de servicios respecto a la base imponible, así mismo son objeto de retenciones del impuesto a la renta aplicada sobre la misma base imponible, estos impuestos deben ser devueltos por la Administración Tributaria en un plazo no mayor a noventa días en el caso de IVA y ciento veinte días laborables en el Impuesto a la Renta, a través de una nota de crédito desmaterializada o transferencia bancaria a la cuenta del contribuyente que hace la solicitud a la Administración tributaria, la cual debe responder oportunamente para que no se generen intereses.

La normativa tributaria, acorde a lo establecido en el Código tributario establece la devolución de impuestos de acuerdo a dos tipos: el pago indebido o el pago en exceso, es decir, los que se haya cancelado sin obligación para hacerlo, o los pagos que hayan sobrepasado el monto correspondiente.

La ley también establece algunos sectores concretos que son vulnerables y requieren de beneficios tributarios, entre ellos el sector florícola. Este proceso de la devolución de impuestos para exportadores se realiza mediante los siguientes pasos:

- Realizar las solicitudes por escrito en la Secretaría del Servicio de Rentas Internas.

- El Servicio de Rentas Interna luego de analizar el trámite con el respectivo tiempo de legitimación, complementación y plazo probatorio de los hechos materia de la solicitud, formula una resolución u oficio para notificar al contribuyente solicitante.

- El Ministerio de Finanzas de acuerdo a la disponibilidad del fondo correspondiente realizará la acreditación.

\section{Devolución del Impuesto al Valor Agregado IVA}

Según el Artículo 51 "El Impuesto al Valor Agregado grava al valor de la trasferencia de dominio o a la importación de bienes muebles de naturaleza corporal, en todas sus etapas de comercialización, así como los derechos de autor de propiedad industrial y derechos conexos; y al valor de los servicios prestados" (Ley Orgánica de Régimen Tributario Interno).

La empresa que genera ventas está obligada a pagar al Servicio de Rentas Internas mediante la declaración mensual en el formulario 104, denominado Declaración del impuesto al valor agregado. De igual manera, para estas empresas se generan costos y gastos generados gravados con tarifa $12 \%$ de IVA, valor que se transforma en un crédito tributario a favor del contribuyente, este valor está sujeto a devolución luego de realizar el trámite respectivo al Servicio de Rentas Internas, por lo que una medida importante que ayudará levantar este sector es la devolución de IVA inmediata. 
El crédito tributario, como su nombre lo indica, es un crédito del que el contribuyente puede hacer uso cuando lo requiera ya sea por devolución o para compensación de futuros impuestos, como diferencia entre el IVA en compras y el IVA en ventas.

Este derecho a un crédito tributario por el IVA se origina si los bienes y servicios comprados se destinan a producir y/o comercializar otros bienes y servicios gravados, o por retenciones de que el contribuyente haya sido objeto. Dependiendo del tipo de transacciones que realice el sujeto pasivo se puede tener: Crédito tributario total, Crédito tributario parcial o Crédito tributario nulo.

En la Figura 1 se aprecia el registro contable, considerando los agentes que intervienen en las transacciones comerciales.

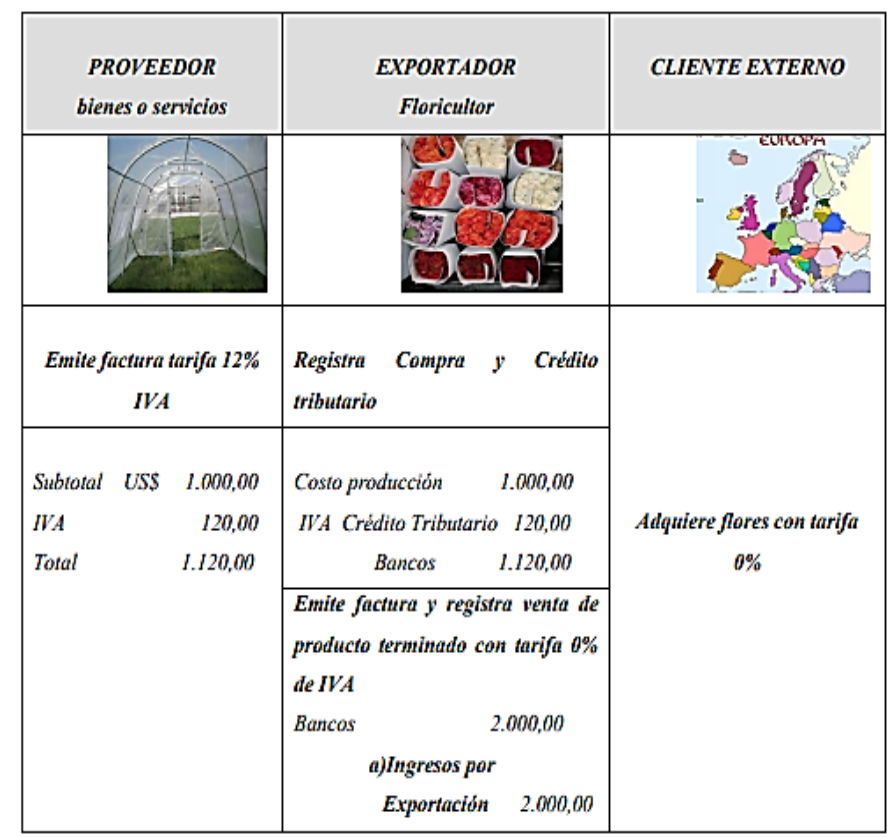

Figura 1. Registro del IVA

Fuente: elaboración propia

De acuerdo al artículo 103 "para sustentar crédito tributario en los pagos por adquisiciones al contado o a crédito, que en conjunto sean superiores a $\$ 1.000$, incluido impuestos, y sean realizadas en beneficio del mismo proveedor o sus partes relacionadas dentro de un mes fiscal, se deberán efectuar a través del Sistema Financiero". (Ley de Régimen Tributario Interno , 2010). Lo que significa que estas transacciones se podrán realizar a través de los instrumentos de pagos tales como giros, transferencias, tarjetas de crédito, débito y cheques.

Tras la publicación en el registro Oficial de la Resolución No. NAC-DGERCGC 19-00000015 publicada en el Suplemento del Registro Oficial No. 462 de 5 de abril de 2019, el gremio de los exportadores podrá acogerse al beneficio impuesto por el Servicio de Rentas Internas, el cual les permitirá solicitar la devolución de IVA de manera inmediata, mismo que según expertos dará una mayor liquidez de las empresas exportadoras. 
Asimismo, todo exportador de bienes y o servicio debe estar registrado con estado activo en el catastro del sistema de devoluciones de IVA por Internet, si es que espera favorecerse de los mecanismos de devolución provisional mediante compensación con retenciones del IVA, por lo que es necesario que se consulte la Norma para ejecutar el procedimiento que más se ajusta de acuerdo al desarrollo de la actividad económica. En la Figura 2 se representan gráficamente los parámetros a los que se sujeta la devolución del IVA a exportadores.

\section{La devolución de IVA a exportadores está sujeta a 4 parámetros:}

Elmenor entre

Crédito tributario mensual (Casillero 602)

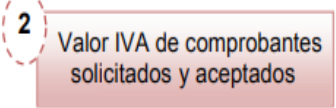

Secompra con

$12 \%$ FOB de las exportaciones aceptadas Crédito acumulado a la
fecha de devolución

En caso de que el menor entre 1,2,3, sea mayor que el valor 4 se genera un saldo a devolver en futuras exportaciones. (Es decir que se recuperará en futuros trámites)

Figura 2. Parámetros para la devolución del IVA a exportadores

Fuente: SRI (s.f)

\section{Devolución del impuesto a la renta IR}

El Impuesto a la Renta, al igual que el IVA, es una obligación tributaria que debe cumplir el contribuyente y por el cual también tiene derecho a solicitar devolución de un saldo a favor si existiese por pago indebido o pago en exceso.

Esta solicitud, también conocida como Reclamo Administrativo de acuerdo al (Artículo 306 del Código Tributario, 2010), que dice: El reclamo administrativo de pago indebido (Artículo 122 del Código Tributario, 2010), y la solicitud de pago en exceso (Artículo 123 del Código Tributario, 2010), se debe presentar ante la autoridad tributaria en los siguientes casos:

- Cuando se ha realizado el pago conforme a un erróneo acto de determinación o de acuerdo a un acta de fiscalización u otro acto, del que no se hubiere presentado reclamo alguno; y,

- Cuando se ha pagado una obligación tributaria inexistente, en todo o en parte, por cuenta propia o ajena. 
Según el artículo 79 para el caso de los contribuyentes que sean sociedades, cuyo plazo de declarar y pagar comienza el 1 de febrero del año siguiente al que corresponda la declaración:

“(...) si no existiese impuesto a la renta causado o si el impuesto causado en el ejercicio corriente fuere inferior al anticipo pagado más las retenciones, el contribuyente tendrá derecho a presentar un reclamo de pago indebido, o una solicitud de pago en exceso, o a utilizar directamente como crédito tributario sin intereses para el pago del impuesto a la renta que cause en los ejercicios impositivos posteriores y hasta dentro de 3 años contados desde la fecha de la declaración." (Reglamento para la Aplicación de la Ley de Régimen Tributario Interno, 2004).

En la Figura 3 se aprecia la plataforma jurídica que sustenta lo concerniente a pagos indebidos o por exceso del impuesto a la renta.

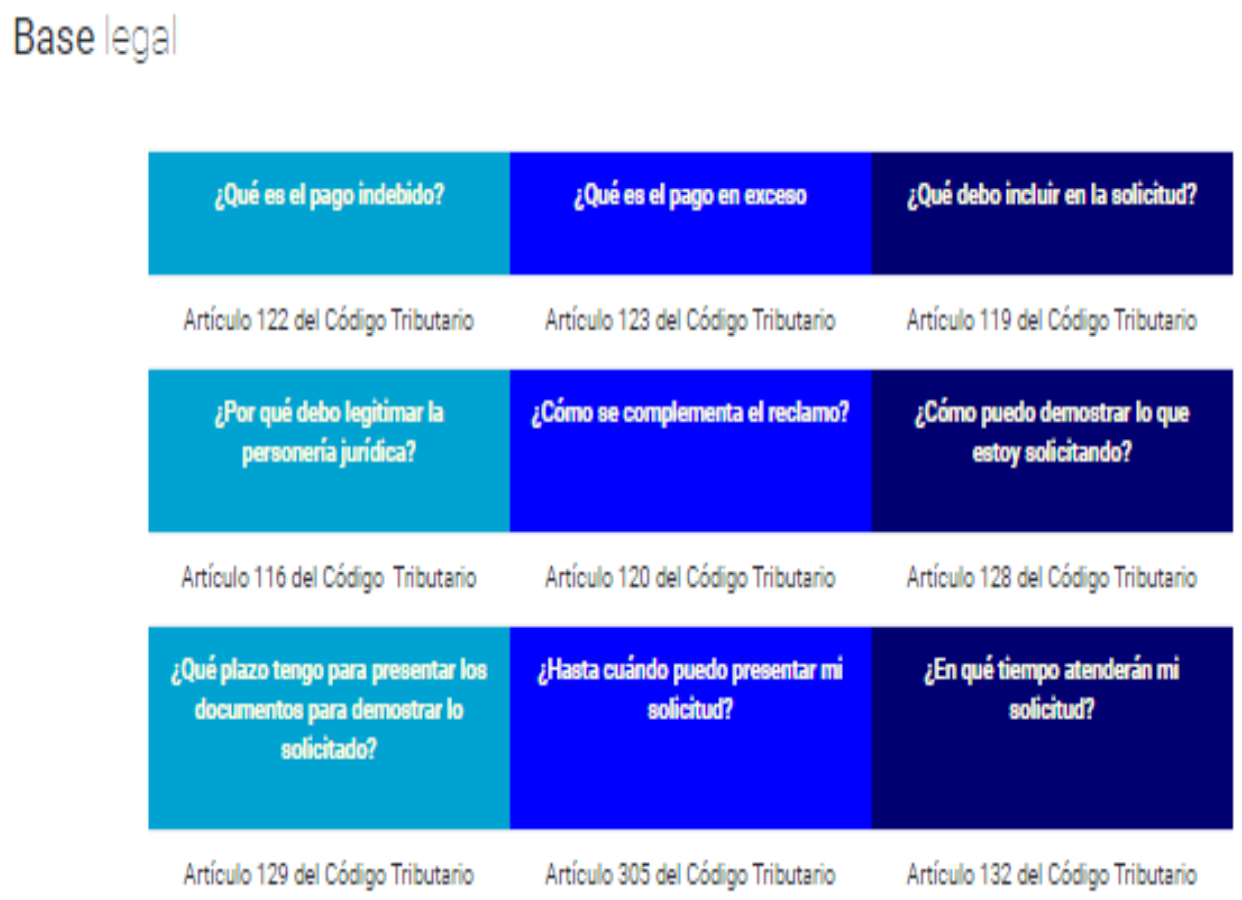

Figura 3. Base legal del pago indebido o en exceso del impuesto a la renta

Fuente: SRI (s.f.)

\section{Metodología}

En cuanto a la metodología, la investigación posee un enfoque cuantitativo, puesto que emplea la recolección de datos numéricos relacionados con la comercialización a nivel internacional de flores, su participación en el Producto Interno Bruto y algunas cifras en cuanto a la situación económica actual del sector, los cuales se analizan acorde a porcentajes y tasas de variación. 
El estudio es de tipo documental ya que emplea fuentes de información contenidas en libros, revistas, leyes, estadísticas y otros. Además, tiene un alcance descriptivo en el cual se identificaron las características más importantes de la producción y comercialización florícola en el Ecuador.

\section{Resultados}

Para evidenciar la importancia del sector florícola en el comercio exterior del Ecuador se presenta en la Tabla 1 la evolución del monto de las exportaciones de flores.

Tabla 1.

Exportaciones del sector florícola (2015-2018)

\begin{tabular}{llllll}
\hline Período & $\begin{array}{l}\text { Total no } \\
\text { tradicionales }\end{array}$ & $\begin{array}{l}\text { Total } \\
\text { Primarios }\end{array}$ & $\begin{array}{l}\text { Flores } \\
\text { naturales }\end{array}$ & $\begin{array}{l}\text { Participación } \\
\text { de sector } \\
\text { florícola en } \\
\text { sector no } \\
\text { tradicional \% }\end{array}$ & $\begin{array}{l}\text { Tasa de } \\
\text { variación \% }\end{array}$ \\
\hline 2015 & 5.365 .890 & 2.094 .414 & 819.939 & 15 & \\
\hline 2016 & 4.881 .232 & 1.664 .961 & 802.461 & 16 & $-2,13$ \\
\hline 2017 & 5.056 .879 & 1.617 .173 & 820.480 & 16 & 2,25 \\
\hline 2018 & 5.241 .086 & 1.705 .305 & 843.372 & 16 & 2,79 \\
\hline
\end{tabular}

Fuente: (Banco Central del Ecuador, s.f.)

"El crecimiento de la producción de flores en Ecuador se inició en la década de los noventas a raíz de que Estados Unidos otorga el ATPDEA a Ecuador, el cual se permitía el ingreso de las flores ecuatorianas con arancel cero a este país" (Corrales Vallejo, 2016).

EI ATPDEA (siglas en inglés para "Andean Trade Promotion and Drug Eradication Act") es el sistema de preferencias comerciales que otorgó Estados Unidos para el acceso libre de aranceles a una gran cantidad de bienes y servicios objeto de las exportaciones, es conocida como la Ley de Preferencias Arancelarias Andinas y Erradicación de la Droga.

Se puede notar en la Tabla 1 que la participación de las exportaciones del sector florícola dentro del total de exportaciones no tradicionales es significativa, puesto alcanza el 15\% en el año 2015 y crece al $16 \%$ en el 2018, lo cual muestra que es un sector robusto dentro de las exportaciones no tradicionales. A su vez, se observa también una alta tasa de variación positiva entre los años 2017 y 2018, exceptuando la variación negativa del año 2015 al 2016. 
Tabla 2.

Ranking de exportadores de flores en el mundo

\begin{tabular}{lll}
\hline Ranking & País & $\begin{array}{l}\text { Participación en las } \\
\text { exportaciones globales }\end{array}$ \\
\hline 1 & Holanda & 48,70 \\
\hline 2 & Colombia & 16,20 \\
\hline 4 & Ecuador & 10,20 \\
\hline 5 & Kenia & 6,30 \\
\hline 6 & Etiopía & 2,30 \\
\hline 8 & Malasia & 1,20 \\
\hline 9 & China & 1,20 \\
\hline 10 & Italia & 1,10 \\
\hline & Bélgica & 1,00 \\
\hline & Alemania & 0,80 \\
\hline
\end{tabular}

Fuente: Ecuador es el tercer exportador mundial de flores (2019)

Tal como se aprecia en la Tabla 2 el sector florícola es de suma importancia para la economía del país, ocupando a nivel mundial el tercer lugar en exportaciones, esto denota el posicionamiento positivo en el mercado internacional con un $10,2 \%$ de participación en el mercado global de flores.

Tabla 3.

Participación del sector florícola en el PIB

\begin{tabular}{llll}
\hline Año & Cultivo de flores & PIB Total & Participación PIB \\
& (MM USD de 2007) & (MM USD de 2007) & \\
\hline 2015 & 504.30 & 70,175 & $0.72 \%$ \\
\hline $2016 \mathrm{sd}$ & 497.82 & 69,314 & $0.72 \%$ \\
\hline $2017 \mathrm{p}$ & 553.29 & 70,956 & $0.78 \%$ \\
\hline $2018 \mathrm{p}$ & 549.00 & 71,871 & $0.76 \%$ \\
\hline sd: semi-definitivo & & & \\
& & & \\
p: provisional & & & \\
\hline
\end{tabular}

Fuente: Corporación Financiera Nacional. Fichas sectoriales 2020

Otra información relevante para expresar la importancia del sector florícola es su participación en el Producto Interno Bruto PIB, en este caso se observa el PIB real, el cual refleja mejor la producción de bienes y servicios, dado que se consideran precios constantes, es decir, se elimina el efecto inflacionario en las cifras. En la Tabla 3 se observa que pasa de 0,72\% en el año 2015 al $0,76 \%$ en el año 2018, lo que implica un aumento del 0,04 \% en sólo tres años. Recalcando que la proporción del aporte al PIB fue mayor en 2017 y sufre una caída de 2 puntos en el 2018. 
Tabla 4.

Empleo generado por cultivo de flores

\begin{tabular}{lll}
\hline Tamaño de la empresa & \# Empresas 2018 & \# Empleados 2018 \\
\hline Grande & 48 & 25502 \\
\hline Mediana & 80 & 9048 \\
\hline Pequeña & 46 & 1225 \\
\hline Microempresa & 63 & 338 \\
\hline Total general & 237 & 36113 \\
\hline
\end{tabular}

Fuente: Corporación Financiera Nacional. Fichas sectoriales 2020

Asimismo, en la Tabla 4 se evidencia la generación de empleos a partir del cultivo de flores, el cual asciende a más de 36 mil empleados, de los cuales el $71 \%$ corresponde a grandes empresas, que en general son las empresas exportadoras del sector.

Tabla 5.

Balance de resultados

\begin{tabular}{lllll}
\hline Cuentas (MM USD) & $\mathbf{2 0 1 5}$ & $\mathbf{2 0 1 6}$ & $\mathbf{2 0 1 7}$ & $\mathbf{2 0 1 8}$ \\
\hline Ingresos & 738.01 & 760.74 & 800.76 & 849.12 \\
\hline Costos y Gastos & 733.17 & 749.52 & 783.33 & 836.12 \\
\hline Utilidad neta & 4.83 & 11.22 & 17.43 & 13.0 \\
\hline
\end{tabular}

Fuente: Corporación Financiera Nacional. Fichas sectoriales 2020

No obstante, el sector respecto a su situación económica refleja cifras que denotan algunas debilidades, tal como se aprecia en la Tabla 5 el crecimiento de beneficios que se venía dando desde 2015 hasta 2017, cae en el 2018 en más de $25 \%$, pasando de 17,43 a 13 millones de dólares.

Tabla 6.

Balance de situación

\begin{tabular}{lllll}
\hline Cuentas (MM USD) & $\mathbf{2 0 1 5}$ & $\mathbf{2 0 1 6}$ & $\mathbf{2 0 1 7}$ & $\mathbf{2 0 1 8}$ \\
\hline Activo & 772.01 & 780.52 & 788.01 & 836.51 \\
\hline Pasivo & 471.75 & 484.64 & 498.64 & 543.18 \\
\hline Patrimonio & 311.71 & 297.05 & 289.37 & 293.32 \\
\hline
\end{tabular}

Fuente: Corporación Financiera Nacional. Fichas sectoriales 2020

Observando el Balance general (Tabla 6) desde el 2015 al 2018 se puede notar que el pasivo se ha incrementado de 471 a 543 millones de dólares, lo cual representa un aumento del $15 \%$ en las obligaciones por pagar, mientras que el activo sólo se incrementa en un poco más del $8 \%$, pasando de 772 a 836 millones de dólares. Si se considera el patrimonio se nota un decremento de 311 a 293 millones de dólares, lo que implica una disminución del 6 \%. Por lo cual se visualiza 
que el pasivo ha ido ascendiendo y contrariamente el patrimonio ha ido descendiendo, lo que implica descapitalización del sector.

Tabla 7.

Impuestos causados por el sector florícola

\begin{tabular}{lllll}
\hline Impuestos (MM USD) & $\mathbf{2 0 1 5}$ & $\mathbf{2 0 1 6}$ & $\mathbf{2 0 1 7}$ & $\mathbf{2 0 1 8}$ \\
\hline IR causado & 4.39 & 6.33 & 8.50 & 8.83 \\
\hline IVA causado & 1.13 & 1.10 & 1.41 & 1.52 \\
\hline Total general & 5.52 & 7.43 & 9.90 & 10.35 \\
\hline
\end{tabular}

Fuente: Corporación Financiera Nacional. Fichas sectoriales 2020

Para finalizar, en la Tabla 7, donde se visualizan los impuestos causados por el sector, se puede destacar del año 2015 al 2018 el incremento del impuesto a la renta IR que pasa de 4,39 a 8,83 millones de USD, lo cual representa un aumento de más del $200 \%$, es decir la cifra se duplica en 3 años. Asimismo, el Impuesto al Valor Agregado IVA pasa del 1,13 a 1,52 millones de dólares, lo que significa un aumento de un poco más del $34 \%$.

\section{Conclusiones}

El sector florícola ha sido muy importante en el comercio exterior del país; si se analiza su estatus para el año 2018 se puede destacar que representa el $16 \%$ del total de exportaciones no tradicionales. Además, se recalca el hecho de que Ecuador ocupa el tercer lugar en el Ranking de exportación de flores a nivel mundial con una participación de un poco más del $10 \%$ del total global. Respecto a su participación en el PIB también es relevante destacar que aporta el 0,76\% del total considerado el PIB real, con tendencia creciente en los años del 2015 al 2018.Además, el sector contribuye significativamente con la economía, puesto que es generador de más de 36 mil empleos.

Pese a esto, para el año 2018 presenta una situación desfavorable cuando se analiza en conjunto su situación económica. Al revisar su estado de resultados se nota una caída del $25 \%$ en sus beneficios, y peor aún al observar su balance general se observa un aumento de los pasivos en 15 $\%$, aunado a un aumento del activo en sólo $8 \%$, lo que conlleva a una disminución del patrimonio del $6 \%$. Esto implica una descapitalización, es decir el agotamiento del capital social, Por otro lado, se observa en cuanto a los impuestos desde el 2015 a I 2018, un aumento que en el caso del IR es del $200 \%$, o sea se duplica y en el caso del IVA aumenta en $34 \%$ aproximadamente.

Aunado a esto, se puede resaltar también el costo de mano de obra, puesto que el salario mínimo en el Ecuador es relativamente alto en comparación con otros de la región, alcanzando en promedio 138 dólares más. Además, existen gastos fijos que incrementan el costo final que no pueden diluirse, por ejemplo, la obligación de tener empleados como médico ocupacional, enfermera, etc. (Florícolas de Ecuador en crisis, 2016).

La baja de los precios internacionales y el descenso de los envíos de flores están siendo influenciados, entre otros factores, a la disminución de la demanda por parte de Rusia que era 
un gran importador. "Esta ha sido uno de los principales destinos de la producción ecuatoriana, pero está inmerso en una batalla comercial-financiera-política con Occidente, que le ha impuesto sanciones y así ha afectado las finanzas rusas" (Los floricultores y la crisis, 2016).

Esta situación conduce a reconocer la necesidad de controlar el flujo de caja para que esta situación no se convierta en un mal endémico que lleve a las empresas del sector a una dificultad que comprometan su competitividad, por lo cual el impacto de la devolución de impuestos indebidos o en exceso en cuanto al IR y del IVA por tratarse de un sector exportador, es de vital importancia dada la necesidad de que se cuente con el cash-flow que permita el funcionamiento de las operaciones y el pago de las obligaciones contraídas, evitando así la descapitalización.

Esta falta de liquidez se agrava dadas las restricciones en el crédito y las fluctuaciones en los precios de negociación. De allí que para el sector sea una problemática explícita y uno de los instrumentos que tiene a mano para mejorar esa situación es la devolución de los impuestos, ya que el impacto de ese reintegro sería la continuidad del sector florícola como uno de los más importantes en el comercio exterior del Ecuador.

Por el pago indebido y pago en exceso el contribuyente puede solicitar la Devolución de Impuesto a la Renta y del IVA a través de un escrito o acción de Petición a la Administración Tributaria Servicio de Rentas Internas. La devolución implica la intervención del Estado, por lo que es el ente de Administración Tributaria quien autoriza el mecanismo y es el Ministerio de Finanzas quien lo acredita.

Dado que las exportaciones de flores van al mercado internacional libres de toda carga impositiva interior con la finalidad de que sus precios solo conciernan a los costos de producción del mercado del país de origen, es decir, no son pechadas con el IVA, los exportadores tienen el derecho a la devolución de este impuesto.

El sector florícola es uno de los exportadores más importantes dentro del grupo de los productos no tradicionales que exporta el país. Las flores se han transformado en un primordial producto de exportación, no obstante, en la actualidad presentan una situación económica dado el decremento de sus beneficios y de su patrimonio, además de las circunstancias adversas que tienen hoy en día por causas tanto endógenas como exógenas.

La situación crítica que afronta el sector por factores internacionales o externos, tales como la contracción de la demanda florícola por parte de Rusia, su principal cliente, la sobreoferta en el mercado internacional y la baja de los precios internacionales y también por un factor interno de mucho peso que es la estructura de costos, en especial el de mano de obra, ya que es más elevado que el costo de países de la región.

Esta problemática ha convergido en falta de liquidez, de allí que para el sector florícola el reembolso de impuestos que en algún momento desembolsó cumpliendo una obligación tributaria, impacta de manera favorable en su liquidez, ya que permite mantener en marcha el proceso productivo y comercial, permitiéndole contar no sólo con el ahorro para inversión, sino para el cumplimiento de las obligaciones. 


\section{Referencias}

Banco Central del Ecuador. (s.f) Exportaciones no tradicionales. [Fichero de datos] https://contenido.bce.fin.ec/ home1/estadisticas/bolmensual/IEMensual.jsp

Código Tributario. (2010). Registro Oficial Suplemento 38 de 14-jun.-2005. Última modificación: 21-ago.-2018

Corporación Financiera Nacional. (2020). Ficha sectorial: cultivo de flores. Fichas sectoriales I Trimestre 2020: https://www.cfn.fin.ec/bibliotecainfo/

Corrales Vallejo, J.M (2016) Análisis del sector florícola ecuatoriano periodo 2005 -2015 caso Cayambe. http:// repositorio.puce.edu.ec/bitstream/handle/22000/12145/tesis\%20final\%20noviembre.pdf?sequence=1\&isAllowed=y. (20012).

Ecuador es el tercer exportador mundial de flores. (2019, febrero 01). Ekos: https://www.ekosnegocios.com/ articulo/ecuador-es-el-tercer-exportador-mundial-de-flores

Florícolas de Ecuador en crisis. (2016, enero 31). La Hora: https://lahora.com.ec/noticia/1101910693/florcolas-de-ecuador-en-crisis.

Ley Orgánica De Régimen Tributario Interno. Decreto Ejecutivo 374 Registro Oficial Suplemento 209 de 08 de junio de 2010. Última modificación: 26 de abril de 2018.

Los floricultores y la crisis. (2016, febrero 05). El Comercio: https://www.elcomercio.com/opinion/editorial/flores-produccion-floricultores-economia-ecuador.html.

Reglamento Para Aplicación De La Ley De Régimen Tributario Interno. Decreto Ejecutivo 374 Registro Oficial Suplemento 209 de 08 de junio de 2010. Última modificación: 26 de abril de 2018.

SRI. (s.f.). Guía rápida proceso de devolución de IVA exportadores. 